(e-migrinter

e-Migrinter

9| 2012

Immigrés, illégaux, réfugiés. Questions sur les enquêtes et les catégories

\title{
Danièle Kergoat ; Adelina Miranda ; Nouria Ouali (dir.), Migrantes et mobilisées
}

\section{Colette Le Petitcorps}

\section{OpenEdition}

\section{Journals}

Édition électronique

URL : https://journals.openedition.org/e-migrinter/788

DOI : 10.4000/e-migrinter.788

ISSN : 1961-9685

Éditeur

UMR 7301 - Migrinter

Édition imprimée

Date de publication : 20 juillet 2012

Pagination : 116-118

ISSN : 1961-9685

Référence électronique

Colette Le Petitcorps, "Danièle Kergoat ; Adelina Miranda ; Nouria Ouali (dir.), Migrantes et mobilisées », e-Migrinter [En ligne], 9 | 2012, mis en ligne le 22 octobre 2018, consulté le 20 mai 2021. URL : http:// journals.openedition.org/e-migrinter/788 ; DOI : https://doi.org/10.4000/e-migrinter.788 


\section{Kergoat, Danièle ; Miranda, Adelina ; Ouali, Nouria (dir.) (2011) Migrantes et mobilisées, Cahiers du gente, $\mathrm{n}^{\circ} 51,265 \mathrm{p}$.}

\section{Colette Le Petitcorps}

$\mathbf{M}$ igrantes d'ancienne date, les femmes ont pourtant longtemps été occultées dans les représentations générales et dans les études scientifiques sur les phénomènes migratoires. Aujourd'hui, nous assistons à un regain d'intérêt vis-à-vis de ces actrices de la mobilité. Ce numéro des Cabiers du Genre coordonné par Danièle Kergoat, Adelina Miranda et Nouria Ouali, interroge tout d'abord le processus de mise en visibilité des femmes migrantes, dans la recherche, dans l'espace public et dans les médias. Au-delà d'une attention médiatique, d'une production scientifique et d'une création artistique décuplées qui mettent en scène la figure de la femme migrante, qu'estce qui est rendu visible? Autour de cette question, les coordinatrices mettent en perspective la manière dont l'articulation entre la cause des femmes, les migrations et une approche culturaliste de celles-ci donne à voir une certaine figure de la femme migrante, victime, dominée par l'homme de sa culture d'origine, et dépendante, tandis que leur rôle de protagonistes de la migration reste dans l'ombre. Le bilan des travaux qui lient femmes, genre et migrations, réalisé par Mirjana Morokvasic, ouvre le débat sur cette mise en visibilité qui, y compris dans le domaine scientifique, "demeure sélective, partielle et partiale », et entrave l'analyse de la complexité des situations migratoires féminines.

Les auteures de ce numéro et ses coordinatrices ont pris le parti de mettre en lumière les formes de mobilisation qui sont à l'initiative des migrantes. Nombreuses, variées et observables sur le temps long, elles ne sont pourtant pas suffisamment explorées. Le terme «migrante » désigne à la fois les migrantes régularisées et sans papiers, les descendantes d'immigré-e-s ou issues des contextes coloniaux. Ainsi, l'ensemble des contributions propose d'examiner le processus par lequel les femmes migrantes s'investissent dans l'action politique et se constituent en sujet politique. Comme le soulignent les différentes chercheuses, l'accès au travail rémunéré des femmes s'est révélé ne pas garantir l'égalité des sexes. L'action politique en revanche, demeure une dimension essentielle du processus d'émancipation.

Différentes manières de décrire le processus de constitution en acteur politique par les femmes migrantes sont mobilisées. Umut Erel décrit la politisation de la position sociale de la femme migrante en partant d'une histoire individuelle et de son prolongement vers la lutte collective en Allemagne. Dans la même perspective, Giovanna Campani démontre comment les trajectoires des migrantes, puis la constitution en association, restent bloquées par les inégalités de genre structurelles à la société italienne. Au lieu de décrire le processus de politisation d'une histoire individuelle à la lutte collective, Hélène Yvonne Meynaud, Helen Schwenken et Cassandra Ellerbe-Dueck retracent l'histoire des mobilisations collectives en France, à l'organisation internationale du travail (OIT), en Allemagne et en Autriche, sans nécessairement passer par le processus d'individuation des femmes migrantes. En outre, un déplacement d'échelles de la 
mobilisation est souligné dans les articles d'Helen Schwenken et de Cassandra EllerbeDueck. Les deux auteures étudient la façon dont des actions politiques partant d'un niveau associatif local sont portées et quelque peu transformées sur la scène internationale et européenne.

L'éclairage de Christine Catarino sur la dimension sexuée des politiques migratoires et des politiques d'emploi vient enrichir ce tour d'horizon des mobilisations des migrantes en Europe, et les différentes échelles de la mobilisation explorées. L'ensemble de ces lectures sur les positions des femmes migrantes en Europe illustre comment, malgré des spécificités nationales contextualisées, les politiques publiques sont intrinsèquement liées à la division sexuelle internationale du travail et maintiennent des inégalités structurelles et postcoloniales qui ont pour terrain de jeu l'espace mondialisé.

Les théories qui croisent les rapports sociaux de genre, de classe et de « race»notamment les théories de l'intersectionnalité ou de la consubstantialité - constituent des outils d'analyse des mobilisations des femmes migrantes. Elles permettent à la fois de comprendre la façon dont le positionnement social des femmes migrantes construit leur subordination, et la manière avec laquelle elles attirent l'attention sur les racismes genrés en se constituant en sujet politique collectif capable de développer un féminisme antiraciste (U. Erel). Pour autant, le genre des migrations et de l'insertion dans le travail n'apparaît pas systématiquement comme un élément à contester dans toutes les mobilisations, comme le montre Helen Schwenken: la reconnaissance de la travailleuse domestique comme travailleuse à l'OIT figure comme revendication principale, laissant de côté la dimension de la division sexuelle du travail. Les mobilisations construisent des identités différentes avec lesquelles il va falloir composer pour unir les féminismes: "femmes migrantes» dans l'exemple d'Umut Erel, "femmes noires» dans l'illustration de Cassandra EllerbeDueck, ou encore «travailleuses domestiques » dans les négociations à l'OIT étudiées par Helen Schwenken.

La portée de l'ouvrage est plurielle. Il propose d'abord une réflexion sur les féminismes, en soulignant les processus de construction des féminismes minoritaires qui permettent de rendre visible différentes formes d'oppression, et de développer des alternatives aux pratiques exclusives de la citoyenneté (U. Erel). La mise en lumière de ces activismes politiques sert à penser, en reflet, l'identité invisible de la «blanchité » inhérente au féminisme dominant. Cependant, cette période de rupture ne serait que transitoire à la mutualisation des luttes pour un féminisme futur. Comme le souligne Giovanna Campani, la condition des femmes migrantes, notamment celle des travailleuses domestiques, est révélatrice de la condition de subordination plus générale des femmes. Ce dossier invite également à une réflexivité sur le positionnement social du ou de la chercheur-e, à l'origine de la construction de l'objet de recherche. La question de la «blanchité» et de la présupposée neutralité scientifique sensibilisent à une déconstruction accrue de nos préjugés et de "ce que l'on veut voir » derrière un phénomène, remettant alors à la surface les convictions idéologiques sousjacentes à la démarche scientifique, et pourtant impensées et effacées du processus de recherche.

Nous terminerons sur un point qu'a souligné Mirjana Morokvasic, à savoir la place centrale qui est conférée aux travailleuses domestiques dans les recherches actuelles sur les femmes et sur le genre en migration. Pour prouver cette affirmation, sur les six articles qui suivent le sien, quatre traitent des travailleuses domestiques. Nous soulevons alors la question: lorsque la 
mobilisation prend appui sur l'identité politique de travailleuse domestique migrante, politisant alors le travail dans l'espace privé, entre-t-elle dans un processus d'émancipation, et peut-elle apporter au féminisme en offrant une nouvelle vision de ce travail ?

Colette Le Petitcorp Doctorante en Sociologie Migrinter - UMR 7301 CNRS / Université de Poitiers lepetit.colette@wanadoo.fr 